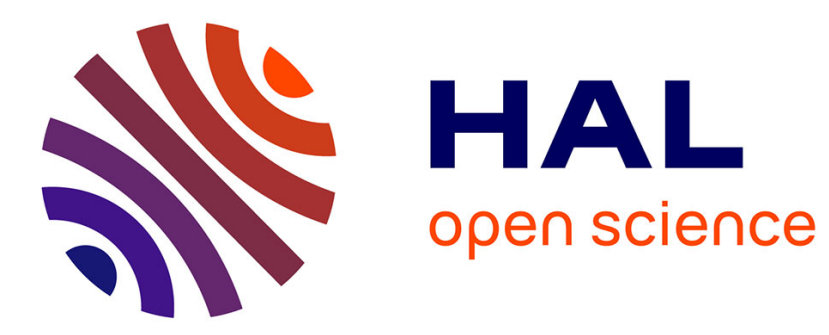

\title{
L'imputation de caractéristiques ethniques dans l'encadrement de la vie scolaire
}

Christian Rinaudo

\section{To cite this version:}

Christian Rinaudo. L'imputation de caractéristiques ethniques dans l'encadrement de la vie scolaire. Revue Europeenne des Migrations Internationales, 1998, 14 (3), pp.27-43. halshs-00080690

\section{HAL Id: halshs-00080690 \\ https://shs.hal.science/halshs-00080690}

Submitted on 20 Jun 2006

HAL is a multi-disciplinary open access archive for the deposit and dissemination of scientific research documents, whether they are published or not. The documents may come from teaching and research institutions in France or abroad, or from public or private research centers.
L'archive ouverte pluridisciplinaire HAL, est destinée au dépôt et à la diffusion de documents scientifiques de niveau recherche, publiés ou non, émanant des établissements d'enseignement et de recherche français ou étrangers, des laboratoires publics ou privés. 


\title{
L’imputation de caractéristiques ethniques dans l'encadrement de la vie scolaire
}

\author{
Christian RINAUDO*
}

(Texte publié dans : Revue Européenne des Migrations Internationales, Vol. 14, n 3, 1998, p. 27-43)

La construction ordinaire des catégories sociales et celle qui relève plus précisément de l'ethnicité a, ces dernières années, fait l’objet de recherches qui ont mis l'accent sur le travail pratique de production des appartenances. Les analyses développées s'inscrivent désormais dans une problématique non substantialiste des identités qui accorde la priorité aux activités de marquage et de maintien des frontières symboliques entre des Nous et des Eux plutôt qu'aux traits culturels qui en constituent les marqueurs ${ }^{1}$.

Les recherches consacrées à l'école constituent à ce jour un cadre privilégié de mise à l'épreuve de ces propositions théoriques même si, comme le soulignent Payet et Van Zanten, poser la question de l'ethnicité dans le contexte français qui fait de cette institution un des piliers de l'intégration des immigrés, reste encore souvent suspect « d’illégitimité républicaine » (Payet et Van Zanten, 1996).

Une des préoccupations des sociologues dans ce domaine aura été d'interroger, à partir de données empiriques, la thèse officielle de "l'école indifférente aux différences ». Leurs travaux ont souligné une surreprésentation des élèves étrangers et d'origine étrangère dans les régions les plus dévalorisées du système scolaire, tant au niveau des filières que des établissements (Payet, 1997). Ils ont montré comment des familles françaises pouvaient être tentées par des pratiques d'évitement des écoles dévalorisées par leur recrutement, à travers le recours au privé ou le détournement de la carte scolaire (Barthon, 1997). Plus encore, ils ont révélé qu'une manière de résister à cette fuite des « bons élèves » consistait, pour les établissements les plus mal cotés, à mettre en place des logiques de ségrégation interne qui se matérialisent par la fabrication de «bonnes » et de «mauvaises classes » (Payet, 1995 ; Debardieux et Tichit, 1997a ; Barrère et Martucelli, 1997). La perception de ces discriminations par les élèves qui en sont victimes a également fait l’objet de nombreux travaux empiriques. Ceux-ci ont montré que leurs expériences scolaires étaient largement surdéterminées par ces logiques discriminatoires (Akers-Porrini et Zirotti, 1992 ; Zirotti, 1997) et que celles-ci favorisaient en retour l'émergence d'une « culture d'opposition » qui s'impose à tous les rapports sociaux et contribue au développement de différentiations ethniques

\footnotetext{
* Université de Nice-Sophia Antipolis ; URMIS-SOLIIS (UMR 7032), Pôle universitaire de Saint-Jean d’Angely, 24, ave des Diables Bleus, 06357 Nice cedex 4.

${ }^{1}$ Ces propositions théoriques ont été présentées dans l'ouvrage de Poutignat et Streiff-Fénart (Poutignat et Streiff-Fénart, 1995).
} 
tant entre les élèves qu'entre les élèves et les adultes (Payet, 1997 ; Debardieux et Tichit, 1997b).

Si toutes ces recherches ont bien montré que les recours à des considérations ethniques étaient à la fois puissants et constants au sein de l'appareil scolaire, elles présentent également ce phénomène comme une forme de « dérapage » de l'institution : alors que l'école républicaine française apparaît communément comme l’un des piliers de l'intégration nationale, elle se trouve dans les faits traversée par des logiques ségrégatives qui remettent en cause le postulat de neutralité culturelle et religieuse sur lequel elle repose. Un tel décalage entre principe et réalité demande toutefois à être interrogé à partir d'autres foyers d'investigation. Car si l'on voit bien la logique de prédiction créatrice par laquelle les « mauvais élèves » en viennent à se construire un univers fortement ethnicisé (les présupposés à leur égard entraînent une stigmatisation, laquelle produit des effets réels qui valident en retour les présupposés de départ), on comprend encore mal comment et pourquoi les enseignants, les surveillants et tous les autres intervenants scolaires (conseillers d'éducation, assistantes sociales...) participent également du processus d'ethnicisation des relations sociales dans les établissements scolaires implantés dans les quartiers populaires.

Notre contribution à l'étude de ce phénomène a été inspirée par une enquête réalisée dans un collège public situé dans un quartier de Nice localement labellisé comme «sensible », «difficile », «à problèmes» depuis la fin des années 70. Totalement absent de l'image officielle de la façade niçoise qui rayonne dans les catalogues touristiques, ce quartier ne constitue pas moins dans toute la région le pôle de référence des quartiers sensibles et le cas d'espèce du problèmes des banlieues tel qu'il se dessine au niveau national. La reconnaissance institutionnelle du caractère problématique de ce quartier a été rendue visible par son inscription incessante depuis plus de quinze ans dans les différents programmes des politiques de la ville: développement social urbain, contrat de ville, zone franche. Il est ainsi au centre d'un faisceau de mesures spécifiques qui, cherchant à améliorer les conditions de vie des habitants dans les différents domaines du champ urbain, participent de sa définition comme « quartier sensible » (Rinaudo, 1998).

\section{UN COLLEGE DE BANLIEUE}

Elevé entre deux grandes cités H.L.M, le collège implanté dans ce quartier est semblable aux milliers d'établissements construits à la hâte dans les années 70 pour faire face à l'extension massive de l'enseignement secondaire. Environ 800 élèves et une soixantaine d'adultes s'y partagent, du lundi au vendredi, un bâtiment de deux étages construit en carré autour d’un patio et d'une vaste cour de récréation. En fonction depuis 1973, il est le seul collège de ce quartier qui, selon la carte scolaire, constitue très exactement son bassin de recrutement. Situé en zone d'éducation prioritaire depuis 1983 et classé «établissement sensible » depuis 1993, il compte, selon les statistiques internes, 59,8 \% d'élèves « issus d’un milieu social défavorisé » et 44,4 \% d’étrangers. 
Notre travail d'observation s'est focalisé sur le domaine particulier que constitue l'encadrement de la vie scolaire : accueil des élèves, prise en charge des retards et des absences, tenue des permanences, surveillance des espaces de récréation et de circulation. C'est en effet à travers toutes ces activités routinières qui rythment la vie d'un établissement que se dévoile le mieux la prise en compte de l'ethnicité par les adultes investis d'un rôle professionnel en milieu scolaire.

Au sein de l'équipe d'encadrement de ce collège, une importance particulière est accordée à la cohésion du groupe et à la solidarité entre ses membres du fait de la spécificité du travail attribuée à l'implantation de l'établissement dans un quartier difficile. Les propos des surveillants sont significatifs de la vigilance érigée en norme face à la violence des rapports sociaux :

« Ici tu ne peux pas te permettre de baisser la garde. On est toujours sur le fil du rasoir et le moindre relâchement de l'attention ne pardonne pas. On doit donc être très soudés entre nous. »

Plus encore, l'ensemble du personnel d'encadrement partage l'idée d'avoir à instaurer un ordre scolaire spécifique, plus adapté à la situation de ce collège. Quelques jours seulement après son recrutement, un surveillant confie ses premières impressions en ces termes :

« Ce qui m'a le plus surpris ici, c'est la violence de tous les enfants. C'est vraiment de la violence sous toutes ses formes. Par exemple, les premières permanences étaient insoutenables. Ils ont fait la fête, ils m'ont... en fait ils m’ont marché dessus. Les jeunes me disaient "viens nous faire une permanence, on va te montrer ce que c'est”. Donc une volonté d'être violent certainement... »

Son étonnement manifeste le sentiment d'évoluer dans un espace social où les codes de conduite ne sont pas les mêmes que dans des établissements dits "normaux », où ce qui vaut pour les autres collèges ne peut être appliqué ici. Cela revient à dire, comme l'a relevé Peralva (1997), que la violence dont il est question dans ce cas échappe radicalement aux canons de la transgression normative. Elle n'est plus envisagée comme une déviance, mais comme un phénomène général qui est renvoyé à une culture partagée par l'ensemble des élèves. Pour un autre surveillant, plus expérimenté que le premier, la nécessité de s' « adapter » à cet environnement passe par la mise en place d'un ordre scolaire négocié, plus adéquat avec la réalité sociale rencontrée, plus en phase avec les valeurs de respect, d'honneur, de virilité qui prévalent dans les collèges de banlieue :

« Il y a une différence très nette dans le niveau de langage entre ici et ailleurs. Ici, lorsque tu surveilles une permanence, si tu dis "tais-toi !” à un élève, c’est comme si tu ne faisais rien. Le minimum, c’est “ferme-la !”. Tout est plus violent qu'ailleurs. Du coup, lorsque tu dois changer d'établissement, tu dois te 
réadapter aux codes normaux. J'ai connu quelqu’un qui a travaillé ici comme surveillant et qui a ensuite été nommé à Antibes. Et bien il a eu des problèmes avec les parents parce qu'il parlait trop crûment aux élèves. Ici, plus rien ne m'étonne. On a eu des élèves qui ont pissé dans le couloir, ça ne m’étonne pas ! C’est comme cracher dans la cour, ici, c’est carrément la norme. Tu n’es même pas étonné par ça. Ailleurs, tu peux mettre un rapport à un élève pour une chose pareille. Ici, tu n’y fais même pas attention, c’est comme ça. »

C'est tout le rapport à la règle et, par extension, à la justice qui est mis en question dans ces propos. En comparant de la sorte l' « ici » et l' «ailleurs », ce surveillant exprime bien l'impossibilité dans laquelle il se trouve d'énoncer la norme et la transgression en tant que phénomène social général, transposable d'un établissement à l'autre. Il ne peut les définir que dans les contextes locaux dans lesquels les faits sont produits et évalués. Cracher dans la cour est perçu comme une transgression à Antibes, pas dans ce collège. Et les sanctions qui sont prises varient en conséquence : ce qui fait l'objet d'un rapport là-bas n'est même pas sanctionné ici. Corrélativement, les modalités d'intervention des surveillants sont soumis à cette même variabilité : ce qui est jugé acceptable, voire même souhaitable ici peut être perçu, par la hiérarchie, par les parents et même par les élèves, comme une transgression dans un autre établissement. On est là en plein cœur de la « crise de l'école » que décrit Dubet, liée aux profondes mutations de la massification scolaire (Dubet, 1994). Le système éducatif français se trouve aujourd'hui dans l'incapacité de fixer des règles applicables en toutes circonstances, ce qui oblige ses intervenants attitrés à les négocier dans les relations interpersonnelles en tenant compte de l'irruption de codes non scolaires dans certains établissements. C’est en tout cas dans ce cadre général que peuvent s'analyser les stratégies de gestion des comportements déviants qui font appel, d'une manière ou d'une autre, à une définition des situations et des individus en termes ethniques.

\section{LES STRATEGIES DE GESTION DE LA VIOLENCE}

Dans leurs relations avec les élèves, les surveillants se défendent d’opérer des distinctions selon les origines culturelles bien que leur mise en saillance soient parfois inévitable dans les situations de la vie scolaire qu'ils doivent affronter et gérer. Un incident qui s'est produit dans l'établissement et qui fut par la suite inscrit dans la mémoire collective de l'équipe montre bien que le risque de voir surgir une définition raciale de la situation reste toujours présent dans le travail d’encadrement.

C'était à l'heure du café, ce petit instant de répit que s'accordent les surveillants entre l'encadrement de la cantine et le retour des externes dans l'établissement. Les demi-pensionnaires vaquaient à leurs occupations dans la cour, foot et basket pour les garçons, badineries sur les starlettes les plus en vogue pour les filles. Mais, comme pour déranger cet état de léthargie dans lequel était plongé le collège, une élève de 6ème vint se plaindre au CPE d'avoir été frappée par un garçon plus âgé qu'elle. Le conseiller d'éducation prévint le père de la jeune élève qui se rendit 
immédiatement sur place, visiblement très en colère. Sa fille lui rapporta l'incident et lui montra du doigt l'auteur de l'agression. S'adressant au surveillant de service, la réaction du père fut des plus violente : « Je suis Sicilien moi, et je ne vais pas me laisser emmerder par des Bougnoules ». Le surveillant réagit promptement et l'entraîna dans un bureau afin que les élèves ne puissent se mêler à l'affaire. Longuement analysée et commentée par la suite, cette scène amena les membres de l'équipe d'encadrement à conclure que «l'altercation aurait pu facilement tourner à l'émeute si les élèves avaient entendu les propos du père de la jeune fille ».

Cet incident brièvement décrit est, comme d'autres moments inscrits dans la mémoire collective de l'équipe, exemplaire des situations de crise où la dimension raciale devient saillante dans la gestion de l'ordre scolaire. Pour y faire face, les surveillants sont souvent amenés à convoquer des compétences pratiques et des connaissances empiriques concernant les différents " groupes ethniques » représentés dans le collège. Renseignements et consignes circulent dans l'établissement et contribuent à établir un "savoir-faire avec ", sorte de code informel des manières d'intervenir en fonction de la spécificité des pratiques culturelles attribuées aux élèves. Par exemple, un surveillant récemment nommé dans l'établissement explique en ces termes comment il fut contraint de prendre en compte certains traits culturels attribués aux élèves gitans et de se méfier de leurs réactions :

« Les Gitans, on m’a carrément averti sur eux. Il faut faire très attention parce que si tu as un problème avec un Gitan, tu as un problème avec tous les Gitans. (...) Ce sont les élèves du collège qui me disent de faire attention aux Gitans... Et encore non, ce sont surtout les surveillants qui m'ont dit de faire attention aux Gitans, de ne pas trop les aborder, de les prendre avec des pincettes, etc. »

De manière générale, on a pu remarquer que la mise en garde sur les comportements des Gitans dans le collège faisait systématiquement partie de l'apprentissage de compétences pratiques dispensé, de manière informelle et empirique, lorsque l'occasion s'en présente, par les membres les plus expérimentés de l'équipe aux nouveaux surveillants. Cet apprentissage passe dans un premier temps par une réification de l'identité gitane autour de traits culturels (l'agressivité, l'imprévisibilité, l'esprit de groupe) envisagés comme une caractéristique première et définitionnelle. Les propos tenus publiquement par un surveillant en poste depuis plusieurs années dans le collège en s'adressant à une nouvelle recrue illustrent bien cette manière de définir les Gitans à partir des caractéristiques culturelles dont ils sont porteurs :

« C’est vrai que bon... tu agis différemment parce que tu sais que les Gitans sont agressifs dans leur manière d'être. En fait ils ne se rendent pas compte qu'ils sont agressifs, pour eux ce n'est pas de l'agressivité. Toi tu vas le percevoir comme de l'agressivité, mais eux ne se sentent pas agressifs. Alors c'est vrai qu'on va laisser dire des trucs parce qu'ils ne se rendent pas compte de ce qu'ils disent. Pour eux c’est normal d'agir comme ça, c’est comme s’ils te parlaient normalement, mais ils le font avec un ton qui te paraît agressif. 
Donc le tout c'est de fixer une certaine limite quand même dans leur manière de parler. »

Dans un second temps, cette socialisation des nouveaux surveillants passe par un enseignement de tous les petits savoir-faire qui, à partir de cette définition substantialisée de l'identité gitane, permet de gérer au mieux les situations de contacts interculturels auxquels les membres de l'équipe sont confrontés en tant que professionnels de l'encadrement de la vie scolaire. Voici comment se terminent les recommandations faites au nouveau surveillant :

« C'est sûr qu'il y a des trucs que tu vas faire avec les Arabes et que tu ne vas pas faire avec les Gitans... Par exemple, tu ne prendras jamais un Gitan par le dos pour lui dire d'aller se ranger parce qu'il se sentirait vraiment agressé. Pourtant ce geste passe facilement chez les Arabes. C’est lié à leur manière de fonctionner. »

La gestion de l'ordre scolaire - comment dois-je agir dans telle situation, comment dois-je me comporter, quelle attitude dois-je adopter, etc. — passe donc par une distinction des élèves en fonction d'inférences sur leurs pratiques culturelles. L'appréhension des élèves est surdéterminée par l'instauration d'un ordre constitué à la fois de la représentation de cultures ethniques supposées bien distinctes, et de la crainte qu'une définition ouvertement raciale de la situation ne devienne saillante dans le déroulement des interactions. Elle consiste à adjoindre un contenu culturel à des catégories ethniques à partir des expériences antérieures, des différentes manières de se comporter et de réagir à telle ou telle situation. Non seulement ce contenu a une fonction descriptive - associé à la catégorie, il sert à lui donner une certaine consistance, un air de réalité empirique qui va de soi («X est un Gitan puisqu’il a la peau mate, les cheveux longs, parce qu'il est agressif, qu'il n’a pas peur des autres ») , mais il détermine aussi une attitude à adopter, une manière de se comporter, une disposition à agir lorsque l'on est en présence d'un membre de cette catégorie : éviter tout contact physique avec un Gitan, ne pas lui mettre la main dans le dos, prévenir l'assistante sociale spécialisée dès que l’on rencontre le moindre problème.

Mais l'ethnicité en tant que phénomène social ne s'exprime pas seulement par l'acquisition de savoir-faire et par la socialisation des surveillants. Elle émerge également dans la mise en œuvre de stratagèmes interactionnels qui s’offrent à eux comme un moyen de négociation des identités sociales et d'imposition de la situation dans le cours des interactions. Ce principe de négociation des identités par les acteurs sociaux a été formulé et étudié par Douglass et Lyman. Ces auteurs soutiennent que l'ethnicité est une caractéristique acquise et utilisable par chaque participant à la vie sociale dans le déroulement de ses interactions. Elle est ainsi sujette à la présentation ou à l'inhibition, à la manipulation et à l'exploitation. Les acteurs sociaux peuvent alors chercher à imposer une définition de la situation leur permettant d'assumer l'identité la 
plus avantageuse dans le cours de l'interaction. Pour cela, ils peuvent mettre en œuvre un certain nombre de choix tactiques, de stratagèmes interactionnels parmi lesquels figurent notamment la présentation d'un moi ethnique le mieux approprié à la situation (assumption), la substitution d'une identité par une autre plus avantageuse dans le cours d'une interaction (identity switching), ou encore l'attribution aux autres interactants d'un rôle ethnique approprié à la définition de la situation que l'on cherche à imposer (alter-casting) (Douglass et Lyman, 1976 ; Lyman et Douglass, 1972).

Deux formes d'utilisation tactique des catégories ethniques sont couramment observées dans l'établissement: la mobilisation d’identités ethniques pour régler les conflits et la valorisation de pratiques culturelles pour lutter contre les comportements déviants.

C'est ainsi par exemple que des rumeurs ont circulé dans le collège sur l'identité corse et les stéréotypes qu'elle véhicule. Il était question d'une tentative de braquage perpétrée contre un véhicule de transport de fonds orchestrée par des " Corses » et impliquant plusieurs membres d'une « famille maghrébine » très connue dans le quartier pour ses activités illicites et les séjours réguliers de ses membres en prison. Cette nouvelle, largement commentée, a contribué à fixer le cadre de la représentation des Corses dans l'établissement, renforçant, par une histoire locale, des stéréotypes déjà fortement marqués par les mouvements nationalistes et par l'image très médiatisée de leurs militants armés et cagoulés.

Quel que soit son bien fondé, cette histoire ne fut pas sans effets dans la relation des membres de l'équipe avec les élèves les plus « durs » du collège ${ }^{2}$. D’abord, parce que la rudesse, l'imprévisibilité et l'inflexibilité attribuées aux «Corses» faisaient des intervenants qui n'étaient pas identifiés et qui ne se définissaient pas euxmêmes comme tels, des acteurs plus facilement manipulables, avec lesquels il devenait plus facile de négocier et d'en imposer. Ensuite, parce que ceux qui affichaient de manière plus ou moins expressive des signaux et emblèmes de cette identité ne niaient pas qu'une telle assumption était parfois utile au bon déroulement des interactions les élèves faisaient plus attention, ils étaient moins grossiers - et devenait de ce fait un véritable choix tactique qui permettait de faire autorité dans le règlement d'un conflit. En d'autres termes, lorsque le seul statut de surveillant ne suffit plus à prendre l'avantage, la mise en saillance d'un moi ethnique à la fois craint et respecté peut être un moyen de conserver l'ascendant dans la relation interpersonnelle. Des formules du type : "Si tu veux qu'on s'explique, c'est d'accord, mais à Ma manière... » ou « Si tu veux ramener ton frère dis-lui bien qu'il vienne armé », théâtralisées par un emphatique accent corse, amènent les élèves récalcitrants à décliner toute manifestation d’hostilité.

\footnotetext{
${ }^{2}$ Durant l'enquête, nous avons rapidement remarqué la présence de nombreux symboles de l'identité corse au sein de l'équipe : panoramas de l’Ile de Beauté affichés dans les bureaux, présence de spécialités culinaires de la même provenance dans la loge des surveillants, allusions à des événements politiques concernant les mouvements nationalistes, etc.
} 
Elles permettent de couper court à toute discussion et de s'imposer frontalement dans l'ordre de l'interaction comme quelqu'un avec qui il vaut mieux ne pas se frotter.

Cette présentation ethnique de soi peut également s’observer dans le règlement de certains conflits comme, par exemple, pour désamorcer une accusation de racisme. Très fréquentes dans les établissements sensibles, les dénonciations de racisme sont pour les élèves une manière de rationaliser leur parcours scolaire et de se protéger de leur échec (Payet, 1992) ou encore, une forme de résistance à un jugement scolaire perçu comme injuste (Dubet et Martuccelli, 1996). Quelle que soit l'interprétation qu'on en donne, elles contraignent ces destinataires (enseignants, conseillers d'éducation, surveillants...) à recourir à des stratégies défensives pour les désamorcer. Un jour où le $\mathrm{CPE}$ du collège reçut dans son bureau une mère d'élève gitane pour lui exposer les problèmes que posait sa fille, il se vit ouvertement accusé de racisme et se trouva dans l'obligation de fournir une réponse acceptable. Non surpris par ce genre d'argument fréquemment avancé par les parents d'élèves gitans, le conseiller d'éducation rétorqua à la mère qu'il n’était pas dans ses habitudes de faire des différences entre les élèves. Et pour argumenter son propos, pour lui donner plus de poids et de crédibilité, il évoqua son identité corse, lui confia qu'il se considérait luimême comme faisant partie d'une minorité et qu'il pouvait de ce fait comprendre son sentiment de révolte et d'indignation, sans pour autant que cela ne l'empêche d'exercer sa fonction de manière impartiale.

Ici, l'assumption a pour but de rendre non recevable une accusation de racisme et de se tirer honorablement d'une situation conflictuelle extrêmement menaçante. Tout comme dans l'exemple précédant, elle est une manière pour les adultes investis d'un rôle professionnel dans l'établissement de se construire une représentation de soi, un self, à partir d'attributs ethniques qui ne sont pas définis par l'institution, sur des principes statutaires, mais, en situation, dans le cadre d'une relation interpersonnelle.

L'autre forme d'utilisation tactique de l'ethnicité consiste, cette fois, à attribuer un rôle ethnique (alter-casting) à des élèves pour enrayer leurs comportements déviants. Les stratégies de gestion de la violence scolaire qui jouent sur des orientations de valeurs associées à un groupe spécifique entrent dans ce cas de figure. Ce qu’on observe pendant le ramadan peut ici servir d'analyseur.

Durant toute cette période, le jeûne devient un objet de discussion banalisé qui fait rapidement partie de l'ordre des choses dans le collège. Il n'est pas rare par exemple qu'un délégué de classe qui vient chercher son cahier de texte dans la loge soit interrogé sur son abstinence. Les surveillants s'inquiètent de la résistance des élèves, ils sont plus indulgents, plus attentionnés. Ils instaurent des relations plus intimes avec eux et peuvent du même coup se voir accepter comme des membres honoraires (Goffman, 1975) avec qui il devient possible d'établir une connivence particulière. Il s’agit là 
d'une compétence relationnelle assez bien maîtrisée par les membres de l'équipe d'encadrement.

Le ramadan fixe également le cadre qui permet de définir les situations et le rythme de la vie scolaire dans le collège. Il sert de grille de lecture du comportement des élèves, de leur agitation, de leur état de fatigue et d'agressivité, de leurs conduites déviantes. Le matin du premier jour de jeûne, le ton fut ainsi donné par le conseiller d'éducation : «Quand je les ai vus rentrer ce matin, je savais qu'ils allaient être calmes. C'est le début du ramadan aujourd'hui ». Quelques jours plus tard, un surveillant ironisa sur le comportement des Gitans en faisant référence au ramadan : « En ce moment, je ne sais pas ce qui se passe avec les Gitans, mais on n'arrive plus à les tenir. Ils sont déchaînés... Je ne sais pas pourquoi, ils ne font pas le ramadan pourtant ».

Mais le ramadan peut également être utilisé comme un moyen de pression pour lutter contre la déviance scolaire. Par exemple, les surveillants rappellent souvent aux élèves qui pratiquent le jeûne que celui-ci ne se limite pas aux seules abstinences alimentaires et sexuelles, mais que certains gestes et comportements peuvent également le rompre. Ainsi, le fait de jurer, de «traiter » ses camarades de classe, de cracher dans les couloirs, de se battre dans la cour de récréation, sont autant d'activités routinières dans le collège qui sont définies, en période de ramadan, comme des actes qui entraînent la rupture du jeûne.

Une scène tout à fait ordinaire de la vie scolaire illustre bien cette pratique. Elle s'est produite à l'heure de la récréation, ce moment de tension extrême où la vigilance de toute l'équipe d'encadrement est à son comble. Deux élèves de Gème qui se taquinaient amicalement dans la cour se mirent à se battre de plus en plus violemment, si bien que le surveillant de service qui les observait de loin s'interposa promptement et entreprit de les raisonner : "Tu te rends compte de ce que tu fais? Tu te bats un jour de ramadan. Ce n'est même plus la peine de continuer maintenant, c'est foutu ! » L'élève interpellé se mit à pleurer : "Mais j'essayais de ne pas me battre pendant le ramadan, c'est de sa faute ». Il montrait du doigt l'autre élève impliqué dans la bagarre qui ne semblait pas être affecté par ces arguments. «Au début on jouait, reprit-il, et après il m’a donné des gifles. Je lui ai demandé d'arrêter mais il a continué ». Le surveillant lui rétorqua que ce n'était pas une raison et qu'il ne devait pas se battre un jour de ramadan, moins encore que les autres jours.

Les incidents ordinaires sont ainsi l'objet d'une reformulation de l'ordre scolaire et le ramadan devient un argument mobilisable pour obtenir des élèves une conformité à laquelle il est difficile de parvenir en temps normal.

\section{UNE STRUCTURE POUR LES ELEVES GITANS}

Etant donnée l'importance accordée aux Gitans par tout le personnel d'encadrement, une de nos premières démarches dans le collège fut de nous renseigner auprès de l'administration et des différentes personnes compétentes sur le nombre 
d'élèves gitans scolarisés. La question n'était pas de chercher à établir des statistiques précises, mais d'évaluer, au moins de manière approximative, pour les besoins de l'enquête, le poids de cette catégorie par rapport à la population totale de l'établissement. Les réponses obtenues à cette requête en disent long sur le procès de catégorisation à l'œuvre lorsqu'il est question des Gitans. On nous parlait d'une vingtaine d'individus, voire d'une trentaine sans plus de rigueur, si bien qu'on entreprit de s'intéresser aux raisons de cette approximation manifeste en demandant aux personnes les mieux informées de dresser, en notre présence, une liste des élèves considérés comme tels. Nous les avons ensuite interrogé sur leurs réponses et nous sommes inquiétés de savoir pourquoi tels et tels noms qui nous furent donnés par ailleurs ne figuraient pas sur leur propre liste. Les remarques recueillies à cette occasion furent très éclairantes: «je n’ai pas parlé d’untel parce qu’il ne me pose aucun problème particulier », " untel est parfaitement intégré et c'est vrai qu’on ne le considère plus comme Gitan », " untel est pour nous un élève comme les autres même si sa famille est Gitane ».

Ainsi, cette attribution catégorielle n'a de sens que par sa mise en relation avec des caractéristiques d' "inassimilabilité culturelle " attribuées à des pratiques et des comportements perturbateurs de l'ordre scolaire. Dès lors, une particularité de la présence de ces élèves qualifiés de "gitans" dans le collège est que le statut d'individus « à problèmes » constitutif de leur visibilité sociale relativise la spécificité des « Maghrébins ». Lorsqu'un surveillant affirme que tous les élèves, "qu’il soient Arabes ou Blancs », ont peur des Gitans, ou quand le CPE explique que « l'insertion est une tâche difficile pour certains élèves d'origine européenne et pour les Maghrébins, mais encore plus dure pour les Gitans », c'est bien cette différence de position qu'ils soulignent. Et la catégorie " arabe » perd du même coup le stigmate social qui lui est généralement attribué dans les banlieues, faisant des «Maghrébins " l'objet d'une représentation plus proche de ce que l'on attend d'un élève « normal » dans un collège. Face aux «Gitans » qui représentent une menace pour l'ordre scolaire, les "Maghrébins » constituent la catégorie modale, celle qui fixe les repères, qui sert de base à la comparaison des comportements déviants et à l'adoption d'une conduite appropriée.

C’est dans ce contexte que les responsables du collège en vinrent à établir une structure d'encadrement pédagogique pour les élèves gitans. Instituée en 1989 sous le titre officiel de « Structure adaptée pour enfants tziganes », son objectif était d'instaurer un dispositif permettant «une prise en compte de la spécificité de la communauté tzigane ${ }^{3}$ et de recueillir tous ceux qui n’étaient pas intégrés au cursus «normal ». Si cette structure fonctionne de manière indépendante - elle dispose d'un personnel

\footnotetext{
${ }^{3}$ Cette initiative a été validée au niveau national par la Direction des Lycées et des Collèges comme « expérience pilote » et dotée d'une subvention du Conseil Général des Alpes-Maritimes. Dans sa configuration actuelle, cette structure est composée d'un personnel spécialisé comprenant une assistante sociale, un éducateur et un enseignant. Elle est placée sous la responsabilité du chef d'établissement et du directeur adjoint chargé de la SEGPA (Section d’enseignement général et professionnel adaptée).
} 
éducatif spécifique et de ses propres salles de classe particulièrement repérables dans l'enceinte de l'établissement —, elle n'est pas conçue comme un dispositif supplémentaire, mais comme un sous-ensemble de la SEGPA du collège destiné à accueillir, parmi les élèves qui relèvent d'un placement dans cette section, ceux qui sont identifiés et reconnus comme des « élèves gitans ».

Les motifs invoqués pour légitimer le traitement différencié des Gitans sont essentiellement d'ordre culturels. Pour les membres de l'équipe d'encadrement, le placement de ces élèves dans une structure spécialisée ne relève pas d'une incapacité intellectuelle à s'adapter au cursus «normal » — ce qui est généralement le cas des élèves placés en SEGPA —, mais plutôt d'une "incompatibilité culturelle » qui les amènerait à se décourager et à s'exclure d'eux-mêmes. Voici par exemple comment un surveillant en poste depuis plusieurs années analyse le passage de Didier, un « élève gitan », du cursus « normal » à la Structure adaptée :

« Moi je l'ai vu avec Didier. Pour moi c’était un cas ce gamin. Il nous a fait la java pire que B., pire que D. (deux autres « cas » connus dans l'établissement), le feu hein, les premiers mois le feu et la classe c'était... je n'aurais pas aimé les avoir en permanence. Et lui il faisait des pieds et des mains pour partir en structure, parce que les Gitans... me retrouver dans le collège normal je ne suis pas un Gitan. Le seul qui y a été c’était L., en 5ème il a foutu le camp il est parti, en 3ème, il était en insertion, il n'est jamais venu en cours. Donc il reste Didier tout seul. Alors en 6ème il est petit, il n'arrive pas encore à comprendre... mais là il a explosé et il est devenu intenable... et depuis qu'il est en Structure, nous on l'a souvent en permanence, il est dix fois moins bordélique qu'il pouvait l'être. Comme s’il avait trouvé un semblant d’identité, c'est ahurissant... Parce que nous, Education nationale, vie scolaire, on pourrait le considérer comme un échec scolaire parce que c'est un gamin qui est suffisamment intelligent pour suivre un cursus normal... »

D’abord, ces propos confirment la relation implicite que nous avons observée entre la catégorie "élèves gitans » et les comportements perturbateurs de l'ordre scolaire. Ils montrent que ceux qui sont identifiés comme tels ont bien intégré l'équivalence qui est faite dans le collège entre cette configuration de sens (Gitans = problème) et la Structure adaptée, et, par une stratégie de retournement du stigmate, transforment cette identité prescrite en une identité souscrite : "me retrouver dans le collège normal je ne suis pas un Gitan ». Mais plus encore, il y a l'idée que l'équilibre des Gitans passe par leur reconnaissance en tant que tels, et que cette reconnaissance s'exprime pleinement lorsque ces élèves sont placés dans une Structure adaptée qui tient compte de leurs spécificités culturelles et qui leur permet d'assumer pleinement cette identité : en 6ème normale, Didier était "intenable ", "bordélique » et depuis qu'il est en Structure... En ses propres termes, le CPE du collège confirme cette théorie culturaliste de sens commun qui fait des Gitans un groupe séparé, " une île coupée du reste du monde » dirait Barth (1995, p. 207), et dont la culture, forcément spécifique, 
que l’on peut légitimement isoler, est présentée comme inconciliable avec les exigences propres à la culture scolaire :

« Même si ce sont des gamins (les Gitans) qui ont un bon niveau scolaire en entrant en 6ème et qui, au vu des résultats qu'ils ont fournis en CM2, pourraient faire de bons élèves de 6ème, ils se trouvent alors complètement déconnectés par le rythme de la 6ème, le changement de profs, le changement de classe, les matières qu'on leur propose, etc. Ils n'arrivent pas à suivre parce qu'ils ne sont pas concernés par tout ça... l'anglais, les sciences, enfin tout ce qu'on peut faire en 6ème. »

Bien que cette « culture gitane » soit décrite comme très différente de ce que l'on peut attendre d'un élève scolarisé dans un cursus dit "normal », elle ne reste pas moins conciliable avec une formation professionnelle spécialisée qui est certes peu valorisée par l'institution scolaire, mais qui est présentée par les différents intervenants de l'établissement comme une véritable promotion pour les élèves gitans compte tenu de leur « handicap culturel ». L'assistante sociale de la Structure adaptée en donne un exemple éclairant :

« On a une gamine maintenant qui travaille, qui a été embauchée à $A$ (une entreprise de restauration industrielle). Lorsqu'elle est rentrée en Structure, c'était une écorchée vive, une gamine avec qui on avait beaucoup de mal à faire du travail, qui avait un niveau épouvantable... En un an il y a eu le déclic, moi je peux dire grâce à la Structure parce que la Structure c'est quand même plus rassurant. Et l'année d'après elle a fait les stages... Maintenant elle va sur ses 18 ans, elle a fait les stages et elle a été embauchée à $A$. Elle a eu un cursus assez intéressant qui est un peu le but de la Structure... »

Ainsi, la Structure adaptée est décrite comme une interface qui se donne comme objectif prioritaire d'acculturer les élèves gitans, du moins, ce qui transparaît bien de cet exemple, dans une certaine mesure. Car si, selon la thèse culturaliste mobilisée, les Gitans ne sont pas "faits » pour des études abstraites (l'anglais, les sciences et tout ce qu'on peut faire en 6ème) et pour le mode d'organisation de l'enseignement secondaire (rythmes soutenus, changements de profs et de salles, etc.), ils peuvent tout de même "s'en sortir " lorsqu'on les oriente vers des filières qui tiennent compte de leurs spécificités et qui, par un enseignement professionnalisant fait de travaux en ateliers, de stages en entreprises et de contrats d'apprentissage, les forment à devenir, comme on dit, de « bons ouvriers ».

Pour expliquer ce cas qui est fait des Gitans, la référence au nomadisme est souvent évoquée, tant par les membres de l'équipe d'encadrement que par le personnel spécialisé de la Structure adaptée. L'absentéisme, le refus de respecter les règles d'usage de la vie scolaire, la volonté de rester entre soi et de ne pas travailler sont autant de traits caractéristiques qui sont attribués à la « culture nomade »: 
« Ici, on dispose d'une structure qui les accueille car c'est une population qui a besoin quand même d'être cadrée, d’être dans un truc bien défini. On a donc essayé de créer une structure pour accueillir ne serait-ce que cinq ou six jeunes au départ, vraiment motivés, qui ont l'air d'avoir envie de s'en sortir parce qu'il faut cette volonté aussi... Car il y en a beaucoup qui se sentent bien comme ils sont, qui n'ont pas envie d'avoir un boulot, qui n'ont pas envie d'avoir un emploi... A la limite, c'est culturel. Ils ont toujours eu la bougeotte. »

Ces jugements de l'éducateur de la Structure adaptée expriment un sens « naturalisé » du nomadisme des Gitans. Même si ceux-ci sont sédentarisés depuis plusieurs générations, même si les élèves en question ont toujours vécu dans ce quartier, il resterait au plus profond d'eux-mêmes cette propension au mouvement qui expliquerait, aujourd'hui encore, leurs difficultés à tenir en place et leur absence de motivation pour le travail sédentaire. La Structure adaptée est alors définie comme un outil permettant de mieux encadrer et de faire évoluer cette population qui a une tendance, culturellement inscrite, à se disperser et à se laisser vivre. L'assistante sociale en charge des élèves gitans dans le collège ne dit pas autre chose lorsqu'elle affirme que ceux-ci sont, comme elle dit, « en apprentissage » :

« Le système scolaire, chez eux, n’est pas encore développé. Ce n’est pas encore vraiment acquis, ça ne fait pas partie de leur culture. La culture tzigane est une culture orale, il n’y a pas d'écrit. Donc pour eux, l'école est quelque chose de nouveau. Ils en sont au «b-a-ba» de la scolarisation. Avant, on ne mettait pas les enfants tziganes à l'école. Donc en quelque sorte ils sont en apprentissage. »

Ses propos illustrent bien la mission civilisatrice que se fixent les éducateurs du collège. Ils montrent également comment ceux-ci arguent de la nécessité d’une Structure adaptée pour les élèves gitans par des attributs culturels (nomadisme, tradition orale des Gitans, absence de contrôle social sur les enfants) considérés comme les caractéristiques premières et définitionnelles de leur organisation en tant que groupe ethnique et, plus encore, en tant qu'altérité négative, à la fois déficiente et dépréciative : «Si les parents décident de ne pas mettre leurs enfants à l'école, explique encore l'assistante sociale, ils ne les mettent pas. Il n’y a pas ce contrôle que l'on peut exercer chez nous ». De sorte que toute conduite ou activité décrite de manière valorisante ou gratifiante est perçue comme le signe d'une évolution, d'une «acculturation », et non comme le propre de l’altérité gitane.

On pourrait appliquer à l'ensemble de cette démarche les critiques formulées à l'encontre des thèses de l'anthropologie culturelle, à savoir que les attributs culturels d'un groupe ethnique ne sont pas des caractéristiques premières et fondamentales, celles à partir desquelles on peut distinguer les membres des non membres, mais un résultat, une implication, soit un ensemble de ressources utilisables par les individus pour marquer les différences entre des Nous et des Eux. Toutefois, notre propos n’était pas 
de démontrer, une fois de plus, les failles logiques du raisonnement culturaliste, mais de rendre compte de la manière par laquelle les différents intervenants concernés en viennent à légitimer, à partir de ces arguments culturels, l'intérêt d'un dispositif qui instaure une ségrégation ethnique au sein même d'un établissement scolaire. Or, cela revient, paradoxalement, à justifier la création d'un "ghetto culturel » pour ne pas exclure les "élèves gitans » d'un système scolaire qui, par principe, ne fait pas de distinctions ethniques. En d'autres termes, il est question d'accepter, au nom de l'intérêt général, le principe de l’intervention de tout un personnel spécialisé (assistante sociale, éducateur, enseignants) dont la tâche consiste à prendre en charge ces élèves et, par là même, à rompre avec l'un des fondements sur lesquels repose l'institution toute entière.

\section{CONCLUSION EN FORME DE PARADOXE}

L'ensemble de ces descriptions montre comment, dans le contexte de crise qui caractérise ce collège (violence des rapports sociaux, impossibilité d'appliquer les codes en vigueur dans les autres établissements) et, par extension, tous ceux qu'avec Payet on a coutume d'appeler les " collèges de banlieue », des catégories ethniques et les inférences qu'elles autorisent participent de la production de grilles de lecture de la réalité qui permettent aux différents intervenants investis de la mission d'encadrement de la vie scolaire de définir le cadre et les modalités de leurs actions. Elles montrent également comment ces catégories constituent des ressources pour interpréter les situations auxquelles ces acteurs sont confrontés en tant que professionnels (incivilités, intimidations, violences verbales et physiques, absentéisme) et comment elles participent de la fabrication de la ségrégation.

Ces analyses nous amènent à conclure que les imputations de caractéristiques ethniques que l'on observe dans les différentes activités qui organisent le travail d'encadrement ne s'opèrent pas tant en dépit du principe d'indifférence aux différences, mais plutôt du fait même de la volonté de maintenir le cadre de l'école républicaine dans le contexte de crise qui caractérise les banlieues. Dans toutes les situations décrites, c'est ce même principe paradoxal qui est à l'œuvre : faire face à l'irruption violente de la dimension raciale en mobilisant des compétences pratiques qui reposent sur une représentation ethnicisée de l'établissement; obtenir, à partir de valeurs ethniques assumées ou attribuées, une normalité sociale à laquelle il est de plus en plus difficile de parvenir par les moyens classiques d'intervention ; valoriser une structure de type ségrégative au nom de l'intégration d'élèves définis d'emblée comme différents. Feldblum arrivait à cette même conclusion lorsqu'elle montrait que les mesures prises dans le but de favoriser l'intégration des jeunes filles d'origine maghrébine s'appuyaient sur une prise en compte institutionnelle de leurs différences culturelles, et soulignait par là qu'une politique non ethnique d'assimilation des immigrés ne contribuait pas moins à faire de l'ethnicité une donnée factuelle, inspiratrice de l'action sociale et politique (Feldblum, 1993). 
Aussi, une interrogation demeure au terme de cette analyse, sur l'intégration et le rôle central attribué à l'école républicaine en France. Dans son étude des logiques de micro-ségrégations scolaires, Payet a surtout insisté sur ce qu'il appelle les « ratés » du processus d'intégration. Il montre en effet que, dans un contexte où l'éthique républicaine est mise à mal par la crise économique et sociale, l'école devient un marché dont les enfants d’immigrés risquent fort de devenir les victimes (Payet, 1997, p. 10). Ce que nous décrivons ici répond à une autre logique : celle, pragmatique, qui consiste à faire des distinctions ethniques pour que soient respectés le mieux possible la paix sociale et l'ordre scolaire dans les établissements sensibles. Cette méthode de " navigation à vue ", comme la qualifie Peralva à propos de la gestion du problème de la violence dans les collèges de banlieue (Peralva, 1997), ne répond donc pas directement à des exigences d'intégration et participe même de la production et de la réification des catégories et des stéréotypes ethniques. Elle témoigne pourtant de la capacité des professionnels de l'encadrement à concilier principes et réalité, puisqu'en faisant de l'ethnicité une ressource mobilisable, non pas pour affirmer et légitimer des différences, mais pour pacifier un ordre scolaire menacé, ils ne contribuent pas moins, à leur manière, à maintenir et à légitimer cette définition non ethnique constitutive de l'école républicaine.

\section{BIBLIOGRAPHIE}

AKERS-PORRINI (R.), ZIROTTI (J.-P.), « Elèves français et maghrébins : un rapport différent à l'orientation scolaire », Migrants-Formation, n 89, 1992.

BARRERE (A.), MARTUCELLI (D.), « L'école à l'épreuve de l'ethnicité », Annales de la recherche urbaine, $\mathrm{n}^{\circ} 75$, juin 1997, pp. 51-58.

BARTH (F.), Les groupes ethniques et leurs frontières, in Théories de l'ethnicité, Paris, P.U.F, 1995.

BARTHON (C.), « Enfants d'immigrés dans la division sociale et scolaire. L'exemple d'Asnières-sur-Seine », Annales de la recherche urbaine, ${ }^{\circ} 75$, juin 1997, pp. 7078.

DEBARDIEUX (E.), TICHIT (L.), « Ethnicité, effet-classe et punition : une étude de cas », Migrants-Formation, n 109, juin 1997a, pp. 138-154.

DEBARDIEUX (E.), TICHIT (L.), " Le construit "ethnique” de la violence ", in Charlot (B.) et Emin (J.-C.) Eds, Violence à l'école. Etat des savoirs, Paris, Armand Colin, 1997b, pp.155-177.

DOUGLASS (W. A.), LYMAN (S. M.), « L'ethnie : structure, processus et saillance », Cahiers internationaux de sociologie, vol. LXI, 1976, pp. 197-220.

DUBET (F.), "Les mutations du système scolaire et les violences à l'école ", Les cahiers de la sécurité intérieure, $\mathrm{n}^{\circ}$ 15, 1994.

DUBET (F.), MARTUCCELLI (D.), A l'école. Sociologie de l'expérience scolaire, Paris, Seuil, L'épreuve des faits, 1996.

FELDBLUM (M.), " Paradoxes of Ethnic Politics : the Case of Franco-Maghrebis in France », Ethnic and Racial Studies, vol. 16, $\mathrm{n}^{\circ}$ 1, January 1993, pp. 52-76. 
GOFFMAN (E.), Stigmate, Les usages sociaux des handicaps, Paris, Editions de Minuit, 1975.

LYMAN (S. M.), DOUGLASS (W. A.), « Ethnicity : Strategies of Collective and Individual Impression Management », Social Research, vol. XL, 1972, pp. 344-365.

PAYET (J.-P.), « Ce que disent les mauvais élèves », Annales de la recherche urbaine, $n^{\circ} 54,1992$.

PAYET (J.-P.), Collèges de banlieue. Ethnographie d'un monde scolaire, Paris, Méridiens Klincksieck, 1995.

PAYET (J.-P.), « La catégorie ethnique dans l'espace des collèges de banlieue : entre censure et soulignement », in Aubert (F.), Tripier (M.) et Vour'ch (F.) Eds, Jeunes issus de l'immigration. De l'école à l'emploi, Paris, CIEMI-L'Harmattan, 1997, pp.207-218.

PAYET (J.-P.), VAN ZANTEN (A.), « L'école, les enfants de l'immigration et des minorités ethniques ", Revue française de pédagogie, $\mathrm{n}^{\circ} 117$, octobre-décembre 1996, pp. 87-149.

PERALVA (A.), " Des collégiens et de la violence », in Charlot (B.) et Emin (J.-C.) Eds, Violence à l'école. Etat des savoirs, Paris, Armand Colin, 1997, pp.101-115.

POUTIGNAT (P.), STREIFF-FENART (J.), Théories de l'ethnicité, Paris, P.U.F, 1995.

RINAUDO (C.), La construction sociale de l'ethnicité en milieu urbain. Production et usages des catégories ethniques dans le cadre d'un quartier "sensible", Thèse de nouveau doctorat de sociologie, Université de Nice-Sophia Antipolis, 1998.

ZIROTTI (J.-P.), « Les enfants immigrés à l'école ou la dynamique de l'altérité », Cahiers de la Méditerranée, vol. 53, nº Juin, 1997.

\section{RESUME :}

Les recherches consacrées à l'école ont souligné jusqu'ici des recours à des considérations ethniques qui pouvaient être analysés comme autant de « dérapages » du modèle laïque et républicain sur lequel repose cette institution. A partir d'une enquête réalisée dans un collège sensible, l'auteur analyse différentes situations dans lesquelles les membres de l'équipe d'encadrement de la vie scolaire sont amenés à procéder à des catégorisations et à mobiliser des stéréotypes ethniques. Il montre alors que ce phénomène ne s’opère pas en dépit du principe d'indifférence aux différences, mais du fait même de la volonté de maintenir le cadre de l'école républicaine dans le contexte de crise qui caractérise les banlieues. En faisant de l'ethnicité une ressource mobilisable pour rétablir un ordre scolaire menacé, ces acteurs ne contribuent pas moins à maintenir et à légitimer une définition non ethnique de l'école.

\section{ABSTRACT :}

Educational researches, up to now, have been focussing on the fact that taking account of ethnicity was a deviant process in regard of the Republican and secular model of the French school. Using an enquiry led in a secondary school considered as raising acute problems, the author analysed several situations in which the members of the team managing the school life are driven to build ethnic categorizations and 
mobilize ethnic stereotypes. According to the author, these phenomenons are not accuring in spite of the official principle of blindness to ethnic differences, but on the contrary because of a will to preserve the Republican frame of the school while coping with the crisis situation characteristic of suburban areas. As ethnicity is promoted as a ressource in order to face threats against the institutional order, educational actors are nevertheless aiming at keeping and legitimizing a non-ethnic definition of the schooling process. 